Прогностическое значение мозгового натрийуретического пептида для оценки риска сердечно-сосудистых осложнений при внесердечных хирургических вмешательствах

\author{
Арапкина О.М.,Ажиоева О.Н. \\ ФГБУ “Национальный медицинский исследовательский центр терапии и профилактической медицины” \\ Минздрава России. Москва, Россия
}

\begin{abstract}
Частота осложнений, связанных с оперативным вмешательством, за последние 30 лет существенно снизилась, однако последствия после хирургических манипуляций все еще являются серьезной клинической проблемой. Выявление интра- и послеоперационного повреждения миокарда является сложной задачей, поскольку почти половина всех осложнений отмечается у пациентов, исходно не входящих в группу высокого риска без заболеваний сердца в анамнезе. В настоящее время несколько крупных исследований и метаанализов, результаты которых были опубликованы за последние 5 лет, рекомендуют использование определения мозговых натрийуретических пептидов для стратификации риска у всех пациентов, готовящихся к плановой внесердечной хирургической операции, с целью улучшения послеоперационного исхода. Мониторинг уровня натрийуретических пептидов у пациентов в течение всего периоперационного периода может способствовать лучшему пониманию патофизиологии развития кардиальных осложнений при внесердечных хирургических вмешательствах. Внедрение новых критериев позволит качественно улучшить оказание помощи комор-
\end{abstract}

бидным пациентам, снизить количество послеоперационных осложнений и улучшить междисциплинарные взаимодействия специалистов в рамках командной работы.

Ключевые слова: внесердечные хирургические вмешательства, мозговой натрийуретический пептид, стратификация сердечнососудистого риска, прогноз послеоперационных осложнений.

Отношения и деятельность: нет.

\section{Поступила 17/04-2020}

Получена рецензия 23/04-2020

Принята к публикации 04/06-2020

\section{(cc) BY 4.0}

Для цитирования: Драпкина О. М., Джиоева О. Н. Прогностическое значение мозгового натрийуретического пептида для оценки риска сердечно-сосудистых осложнений при внесердечных хирургических вмешательствах. Кардиоваскулярная терапия и профилактика. 2020;19(5):2558. doi:10.15829/1728-8800-2020-2558

\title{
Predictive value of brain natriuretic peptide for cardiovascular risk assessment in non-cardiac surgery
}

Drapkina O. M., Dzhioeva O. N.

National Medical Research Center for Therapy and Preventive Medicine. Moscow, Russia

The incidence of postoperative complications has decreased significantly over the past 30 years, but the consequences after surgery are still a serious clinical problem. Identifying intra- and postoperative myocardial injury is a challenging task, since almost half of all complications occur in patients who were not initially at high risk without a history of heart disease. Currently, several large studies and meta-analyzes over the past 5 years recommend the determining brain natriuretic peptide (BNP) levels for risk stratification in all patients scheduled for elective non-cardiac surgery in order to improve the postoperative outcome. Monitoring of BNP level during the entire perioperative period can contribute to a better understanding of the pathophysiology of cardiac complications in noncardiac surgery. The introduction of new criteria will qualitatively improve care to patients with comorbidities, reduce the number of postoperative complications and improve interdisciplinary interactions of specialists.

Key words: non-cardaic surgery, brain natriuretic peptide, cardiovascular risk stratification, prognosis of postoperative complications.
Relationships and Activities: none.

Drapkina O. M. ORCID: 0000-0002-4453-8430, Dzhioeva O. N. * ORCID: 0000-0002-5384-3795

${ }^{*}$ Corresponding author: dzhioevaon@gmail.com

Received: $17 / 04-2020$

Revision Received: 23/04-2020

Accepted: 04/06-2020

For citation: Drapkina O.M., Dzhioeva O.N. Predictive value of brain natriuretic peptide for cardiovascular risk assessment in non-cardiac surgery. Cardiovascular Therapy and Prevention. 2020;19(5):2558. (In Russ.) doi:10.15829/1728-8800-2020-2558

ДИ - доверительный интервал, ОР - относительный риск, ПОФП - послеоперационная фибрилляция предсердий, СН - сердечная недостаточность, СНнФВ - сердечная недостаточность с низкой фракцией выброса, СНсФВ - сердечная недостаточность с сохраненной фракцией выброса, ССЗ - сердечно-сосудистые заболевания, ССО - сердечно-сосудистые осложнения, ФВ - фракция выброса, ФП фибрилляция предсердий, ЭКГ - электрокардиограмма, BNP - мозговой натрийуретический пептид, NT-proBNP - N-концевой фрагмент мозгового натрийуретического пропептида, RCRI - Revised Cardiac Risk Index (пересмотренный индекс сердечного риска).

*Автор, ответственный за переписку (Corresponding author):

e-mail: dzhioevaon@gmail.com

Тел.: +7 (916) 614-18-21

[Драпкина О. М. - д.м.н., профессор, член-корр. РАН, директор, ORCID: 0000-0002-4453-8430, Джиоева О. Н. ${ }^{\star}$ - к. М.н., с.н.с. отдела фундаментальных и прикладных аспектов ожирения, ОRCID: $0000-0002-5384-3795]$ 


\section{Введение}

Особенности ведения пациентов с сердечнососудистыми заболеваниями (СС3) при внесердечных хирургических вмешательствах остаются темой профессиональных дебатов среди специалистов хирургического, терапевтического и анестезиологического профиля. В последние годы общая тенденция в профессиональных обществах заключалась в том, как максимально точно выявлять до операции пациентов с высоким риском осложнений после оперативных вмешательств. Важной и дискутабельной остается проблема целесообразности тех или иных методов предоперационного обследования. Несмотря на то, что частота осложнений, связанных с оперативным вмешательством, за последние 30 лет существенно снизилась, последствия после хирургических манипуляций все еще являются серьезной клинической проблемой. Выявление интра- и послеоперационного повреждения миокарда является сложной задачей, поскольку почти половина всех осложнений отмечается у пациентов, исходно не входящих в группу высокого риска без заболеваний сердца в анамнезе.

В последнее время в качестве новых чувствительных и специфических маркеров международные рекомендации по профилактике сердечно-сосудистых осложнений (ССО) после хирургических вмешательств предлагают использовать мозговой натрийуретический пептид (BNP) или $\mathrm{N}$-концевой фрагмент proBNP (NT-proBNP), особенно у пациентов с высоким риском, подвергающихся внесердечным оперативным вмешательствам [1-3]. Однако в рекомендациях различных профессиональных сообществ также есть противоречия: некоторые специалисты предлагают использование определения натрийуретических пептидов В-типа для стратификации риска у всех пациентов, готовящихся к плановой операции, вне зависимости от степени риска, с целью улучшения послеоперационного исхода, тогда как другие специалисты предлагают использовать этот маркер исключительно у пациентов с предполагаемым высоким риском ССО [3, 4].

Современные рекомендации по оценке сердечно-сосудистого риска и ведению пациентов хирургического профиля традиционно основаны на исключении активных или латентных заболеваний сердца, определении риска операции, функциональных возможностей пациента и наличия факторов риска ССЗ. За последние два десятилетия получены новые доказательства, продемонстрировавшие связь между специфическими биомаркерами и неблагоприятными сердечно-сосудистыми событиями, что открывает новые перспективы в периоперационной профилактической медицине. В настоящее время биомаркеры повреждения миокарда являются одним из основных диагностических критериев сердечной недостаточности $(\mathrm{CH})$. Тем не менее, согласно руководствам Американского кардиологического общества и Европейского общества кардиологов, они пока имеют второстепенное значение при периоперационной оценке сердечно-сосудистого риска $[1,2]$. Канадским обществом кардиологов был предложен алгоритм, включающий определение концентрации лабораторных показателей, обозначив предоперационную оценку BNP или NT-proBNP как основной критерий в системе стратификации риска [3]. Европейское общество анестезиологов также рекомендует использовать натрийуретический пептид В-типа для независимого послеоперационного прогноза у пациентов высокого сердечно-сосудистого риска, подвергающихся внесердечным хирургическим вмешательствам [4]. Как BNP, так и NT-proBNP образуются из proBNP, который синтезируется кардиомиоцитами и фибробластами в ответ на повышение давления наполнения желудочков. Впоследствии этот пептид разрушается ферментом эндопептидазой с периодом полураспада 5-10 мин [5]. ВNР обладает симпатоингибирующим действием и снижает секрецию ренина, ангиотензина и альдостерона. BNP также снижает артериальное давление, увеличивает диурез и обладает вазодилатирующим эффектом. Мозговой натрийуретический пептид был признан лучшим среди всех биомаркеров, используемых для оценки риска ССЗ у пациентов с ранее не диагностированными заболеваниями сердца [6, 7]. У нехирургических пациентов натрийуретический пептид давно включен в диагностические алгоритмы и используется, в основном, для диагностики и оценки тяжести СН [5]. Возможность применения этого маркера у пациентов перед внесердечными операциями стала рассматриваться после анализа результатов исследования VISION (Vascular events In noncardiac Surgery patIents cOhort evaluatioN), в котором участвовало $>10$ тыс. пациентов и в котором была показана сильная корреляционная связь между уровнями BNP и NT-proBNP до операции с неблагоприятными сердечно-сосудистыми событиями в послеоперационном периоде [3]. Предоперационная диагностическая ценность BNP в качестве мощного независимого предиктора краткосрочных ССО при внесердечных операциях в последующем была подтверждена в двух крупных метаанализах [8, 9]. Повышенный уровень BNP у пациентов, определенный перед оперативным вмешательством, был сопряжен с увеличением относительного риска в 17 раз в модели прогнозирования ССО [10]. При попытке обобщения данных проведенных исследований оптимальное пороговое значение для BNP и NT-proBNP у хирургических пациентов из-за большого различия между исследованиями окончательно определить не удалось $[8,9]$. Предполагается, что пороговое значение BNP для прогнозирования сердечной смерти и нефатального инфаркта 
миокарда в течение 30 сут. после внесердечной операции составляет 30 пг/мл с чувствительностью $95 \%$ и специфичностью 44\% [11]. Эти данные вполне согласуются с результатами большинства исследований, в которых изучалась возможность оценки BNP для прогнозирования сердечно-сосудистых событий у бессимптомных пациентов. По пороговому уровню NT-proBNP также нет однозначного мнения, поскольку уровень этого маркера тесно связан с возрастом пациентов. Примечательно, что в продолжающемся исследовании POISE-3 (PeriOperative ISchemic Evaluation-3) предложено к использованию предоперационное значение NT-proBNP $\geqslant 200$ нг/л в качестве одного из критериев включения, указывающих на риск ССО [12]. На основании всех имеющихся данных по проблеме в 2018г был опубликован “Швейцарский алгоритм” от имени Швейцарского общества анестезиологии и реанимации [13]. В соответствии с этим согласительным документом, если подразумевается высокий риск хирургической манипуляции, у пациента выявлены низкие функциональные резервы и определено наличие дополнительных соматических факторов риска, определение уровня BNP целесообразно для дальнейшей прогностической оценки и определения тактики ведения пациента. Пациентам с уровнями биомаркера в пределах нормы хирургическое вмешательство проводится без дополнительного тестирования, в то время как пациентам с повышенным уровнем BNP или NT-proBNP целесообразно провести дополнительное кардиовизуализирующее и функциональное обследование [13].

Согласно современным международным позициям, однократно выявленный повышенный уровень BNP до операции уже является предиктором потенциальных послеоперационных ССО [14]. BNP является мощным прогностическим критерием неблагоприятных исходов у пациентов с любой формой СН [15-19]. У пациентов с диагностированной $\mathrm{CH}$, вне зависимости от фракции выброса (ФВ), увеличение концентрации натрийуретических пептидов на каждые 100 пг/мл было связано с 35\%-ным увеличением относительного риска смерти [19]. Результаты больших проспективных когортных исследований показывают, что уже незначительное повышение BNP может отражать ранние бессимптомные стадии патологических процессов [20]. Вероятно, повышение BNP позволяет выявить пациентов с бессимптомными нарушениями миокардиальной функции, которые входят в группу риска при эндотрахеальной анестезии и крупных хирургических вмешательствах. И чувствительность, и специфичность натрийуретических пептидов в прогнозировании ССО при внесердечных операциях составили $87 \%$ [21]. Стандартно используемый пересмотренный индекс сердечного риска Revised Cardiac Risk Index (RCRI), который включает ишемическую бо- лезнь сердца, $\mathrm{CH}$, сахарный диабет на инсулине, цереброваскулярную болезнь, почечную недостаточность с уровнем креатинина $>2$ мг/дл в качестве 5 независимых предикторов с оценкой от 0 до 5 на основе оценки числа факторов риска, обладал чувствительностью $64 \%$ и специфичностью $70 \%$ [22]. Согласно данным нескольких сравнительных исследований по определению BNP или NT-proBNP и RCRI, предоперационная оценка BNP показала лучшую чувствительность, чем RCRI, в прогнозировании периоперационных ССО [23, 24]. Поскольку было показано, что чувствительность и специфичность BNP лучше, чем у RCRI, было бы целесообразно использовать у предоперационных пациентов оба метода, поскольку клиническую шкалу дополнил бы прогностически значимый лабораторный маркер [25-27]. На основании полученных результатов можно сказать, что в настоящий момент BNP предоставляет максимально точную прогностическую информацию о неблагоприятных исходах вследствие CCO. В то же время, NT-proBNP не является предиктором смерти или сердечно-сосудистых событий у пациентов без традиционных клинических факторов риска ССЗ и эхокардиографических признаков ремоделирования сердца [28]. Определение уровня BNP вряд ли будет экономически эффективным у пациентов с RCRI, равным нулю, в аспекте исходного низкого риска у этих пациентов. Поскольку стоимость теста BNP в США сопоставима со стоимостью выполнения и интерпретации электрокардиограммы (ЭКГ), анализ ВNP в этой стране рассматривается как альтернатива инструментальным методам [8]. Средняя стоимость лабораторной оценки натрийуретических пептидов в России в 5 раз превышает стоимость ЭКГ, поэтому широкое внедрение этого метода ограничено экономической составляющей, а рутинное его использование у всех пациентов перед внесердечным оперативным вмешательством дискутабельно.

Фибрилляция предсердий (ФП) является наиболее распространенным нарушением сердечного ритма, и, хотя сама по себе эта аритмия не является жизнеугрожающей, она увеличивает риск ишемического инсульта у пациента в 5 раз [30]. ФП в послеоперационном периоде связана как с наличием СС3, так и с внесердечными факторами, обусловленными хирургическим стрессом [31]. Очень часто выявление ФП у пациентов после оперативного вмешательства связано с непрерывным стационарным мониторингом сердечного ритма, а сама по себе аритмия является бессимптомной [32]. Однако появляется все больше данных о том, что даже короткие эпизоды послеоперационной ФП (ПОФП) являются значимыми факторами сердечно-сосудистого риска после внесердечных оперативных вмешательств. [33, 34]. ПОФП имеет множество негативных последствий, включая гемодинамическую 
нестабильность и острую $\mathrm{CH}$, а также тромбоэмболические осложнения [35].

Существует несколько потенциальных биомаркеров, позволяющих прогнозировать риск развития ПОФП [36]. Например, маркеры синтеза коллагена (семейство проколлагена), ремоделирования внеклеточного матрикса, воспаления и профибротические медиаторы (интерлейкины) являются перспективными для стратификации потенциального риска развития аритмии. В частности, вариации уровней этих маркеров, как сообщается в литературе, могут отражать различия в методологии исследований, в характеристиках обследуемых групп, типе ФП, наличии дисфункции левого желудочка, а также последствия медикаментозной терапии. В настоящее время натрийуретические пептиды активно изучаются в качестве маркеров ПОФП [36-39]. Недавний систематический обзор оценил, являются ли предоперационные повышение BNP независимым предиктором ФП после операции на грудной клетке [36]. Были проанализированы результаты 5 наблюдательных исследований, которые в последующем были объединены в метаанализ. Повышенные предоперационные уровни BNP были связаны с повышением риска развития ПОФП (относительный риск (ОР) 3,13; $95 \%$ доверительный интервал (ДИ): 1,38-7,12) [37]. Данные другого метаанализа показали, что определение BNP и NT-proBNP у пациентов после операции существенно повышает чувствительность шкалы оценки риска по сравнению с определением только до операции. Cardinale D, et al. показали, что повышенный после операции уровень NT-proBNP предсказывал ПОФП у пациентов, перенесших операцию на легких [38]. Как дооперационные, так и послеоперационные значения NT-proBNP были независимыми предикторами ФП при многофакторном анализе (ОР 27,9; $95 \%$ ДИ: 13,2-58,9; $<0$,001 для предоперационного повышения NT-proBNP; OP 20,1; 95\% ДИ: 5,8-69,4; $p<0,001$ для послеоперационного повышения NTproBNP). Эти результаты были подтверждены в рандомизированном исследовании, проведенном той же группой ученых [29]. В это исследование были включены пациенты с высоким значением NT-proBNP $(\mathrm{n}=1116)$. Профилактическая стратегия с использованием медикаментозной терапии (метопролола или лозартана) у пациентов с повышенной концентрацией NT-proBNP снижала частоту возникновения ПОФП [39]. Более низкая частота развития ПОФП подразумевала сокращение продолжительности госпитализации и связанное с этим меньшее количество послеоперационных нежелательных явлений. Многие опубликованные данные иллюстрируют дополнительную прогностическую роль и важное значение определения уровня BNP в качестве предиктора ПОФП, как в кардиохирургической практике, так и в экстракардиальной хирургии [40-44].
У лиц без значимых СС3 в анамнезе, повышенный уровень натрийуретических пептидов также является предиктором развития ПОФП [43]. Таким образом, остается открытым вопрос: может ли ФП возникать у пациентов без сердечно-сосудистой патологии на фоне исключительно хирургического стресса, или же повышение концентрации натрийуретического пептида, как маркера доклинической стадии повреждения миокарда, свидетельствует об обратном. В этом аспекте важно упомянуть о трудностях диагностики СН у пациентов с сохраненной ФВ (СНсФВ). Несмотря на то, что определение концентрации натрийуретических пептидов является обязательным для постановки диагноза $\mathrm{CH}$, точная роль BNP и NT-proBNP в клиническом фенотипировании при СНсФВ изучена меньше, чем в группах пациентов с СН с промежуточной и низкой ФВ. Это отчасти связано с несоответствиями, ассоциированными с разными диагностическими инструментальными подходами у таких больных, а также с тем фактом, что конечное диастолическое напряжение в стенке, как пусковой механизм для высвобождения BNP/NT-proBNP, ниже при СНcФB, чем у пациентов с низкой ФВ. Кроме того, СНсФВ является гетерогенным клиническим синдромом, который характеризуется сердечно-сосудистыми, метаболическими и провоспалительными изменениями. Поэтому, именно в группе пациентов с сохраненной ФВ возникает неопределенность в отношении роли натрийуретических пептидов в патофизиологии СН. Именно поэтому, у пациентов, подвергающихся хирургическим вмешательствам, важно проводить оценку концентрации биомаркера как в предоперационном периоде, так и после хирургического вмешательства. Мониторинг уровня натрий-уретических пептидов у пациентов в течение всего периоперационного периода может способствовать лучшему пониманию патофизиологии развития ССО при внесердечных хирургических вмешательствах.

В настоящее время рутинный мониторинг уровня натрийуретических пептидов в течение периоперационного периода у пациентов при внесердечных хирургических вмешательствах не включен в алгоритмы действий междисциплинарной врачебной команды. Однако данные все большего числа исследований подтверждают прогностическую ценность этого маркера относительно риска развития $\mathrm{CCO}$, таких как острая $\mathrm{CH}$ или ФП, даже у пациентов без предшествуюшего анамнеза ССЗ. Необходимо проведение большего количества исследований среди пациентов, которым предстоит внесердечное хирургическое вмешательство, по изучению дополнительных диагностических и прогностических значений натрийуретических пептидов. Определение новых критериев позволит оптимизировать междисциплинарные взаимодействия специалистов 
в рамках командной работы, снизить количество послеоперационных осложнений и качественно улучшить оказание помощи коморбидным пациентам.

\section{Литература/References}

1. Fleisher LA, Fleischmann KE, Auerbach AD, et al. 2014 ACC/AHA guideline on perioperative cardiovascular evaluation and management of patients undergoing noncardiac surgery: A report of the American college of cardiology/American heart association task force on practice guidelines. J Am Coll Cardiol. 2014;64:e77-137. doi:10.1016/j.jacc.2014.07.944.

2. Kristensen SD, Knuuti J, Saraste A, et al. 2014 ESC/ESA Guidelines on non-cardiac surgery: cardiovascular assessment and management: the Joint Task Force on non-cardiac surgery: cardiovascular assessment and management of the European Society of Cardiology (ESC) and the European Society of Anaesthesiology (ESA). Eur Heart J. 2014;35:2383-431. doi:10.1097/EJA.0000000000000150.

3. Duceppe E, Parlow J, MacDonald P, et al. Canadian Cardiovascular Society Guidelines on Perioperative Cardiac Risk Assessment and Management for Patients Who Undergo Noncardiac Surgery. Can J Cardiol. 2017;33(1):17-32. doi:10.1016/j.cjca.2016.09.008.

4. De Hert S, Staender S, Fritsch G, et al. Pre-operative evaluation of adults undergoing elective noncardiac surgery: Updated guideline from the European Society of Anaesthesiology. Eur J Anaesthesiol. 2018;35:407-65. doi:10.1097/EJA.0000000000000817.

5. Porapakkham P, Porapakkham P, Zimmet H, et al. B-type natriuretic peptide-guided heart failure therapy: A metaanalysis. Arch Intern Med. 2010;170(6):507-14. doi:10.1001/ archinternmed.2010.35

6. Blankenberg S, Zeller T, Saarela O, et al. Contribution of 30 biomarkers to 10-year cardiovascular risk estimation in 2 population cohorts: the MONICA, risk, genetics, archiving, and monograph (MORGAM) biomarker project. Circulation 2010;121:2388-97. doi:10.1161/CIRCULATIONAHA.109.901413.

7. Wang TJ, Gona P, Larson MG, et al. Multiple biomarkers for the prediction of first major cardiovascular events and death N Engl J Med. 2006;355:2631-9. doi:10.1007/s12170-0090021-z.

8. Karthikeyan G, Moncur RA, Levine O, et al. Is a pre-operative brain natriuretic peptide or $\mathrm{N}$-terminal pro-B-type natriuretic peptide measurement an independent predictor of adverse cardiovascular outcomes within 30 days of noncardiac surgery? A systematic review and meta-analysis of observational studies. J Am Coll Cardiol. 2009;54:1599-606. doi:10.1016/j. jacc.2013.08.1630.

9. Ryding $A D$, Kumar $S$, Worthington $A M$, et al. Prognostic value of brain natriuretic peptide in noncardiac surgery: a meta-analysis. Anesthesiol. 2009;111:311-19. doi:10.1097/ ALN.0b013e3181aaeb11.

10. Rodseth RN, Padayachee L, Biccard BM. A meta-analysis of the utility of pre-operative brain natriuretic peptide in predicting early and intermediate-term mortality and major adverse cardiac events in vascular surgical patients. Anaesthesia. 2008;63:122633. doi:10.1111/j.1365-2044.2008.05574.x.

11. Rodseth RN, Lurati Buse GA, Bolliger D, et al. The predictive ability of pre-operative B-type natriuretic peptide in vascular patients for major adverse cardiac events: an individual patient data meta-analysis. J Am Coll Cardiol. 2011;58:522-9. doi:10.1016/j. jacc.2011.04.018.
Отношения и деятельность: авторы заявляют об отсутствии потенциального конфликта интересов, требующего раскрытия в данной статье.

12. PeriOperative ISchemic Evaluation-3 Trial. https://clinicaltrials. gov/ct2/show/NCT03505723.

13. Filipovic $\mathrm{M}$, Kindler $\mathrm{CH}$, Walder $\mathrm{B}$. Anästhesiologie und Reanimation: Perioperative kardiale Abklärung und Therapie im Vorfeld nicht-herzchirurgischer Eingriffe. Swiss Med. Forum Schweiz. Med. 2018;18:1078-80. doi:10.4414/smf.2018.03440.

14. Biccard BM, Naidoo P, De Vasconcellos $K$. What is the best pre-operative risk stratification tool for major adverse cardiac events following elective vascular surgery? A prospective observational cohort study evaluating pre-operative myocardial ischaemia monitoring and biomarker analysis. Anaesthesia. 2012;67:389-95. doi:10.1111/j.1365-2044.2011.07020.x.

15. Shimizu Y, Nishinaga M, Takata J, et al. B-type natriuretic peptide is predictive of hospitalization in community-dwelling elderly without heart diseases. Geriatr Gerontol Int. 2009;9:148-54. doi:10.1186/2049-6958-9-59.

16. Wang TJ, Larson MG, Levy D, et al. Plasma natriuretic peptide levels and the risk of cardiovascular events and death. N Engl J Med. 2004;350:655-63. doi:10.1056/NEJMoa031994.

17. Witham MD, Gillespie ND, Hutcheon SD, et al. B-type natriuretic peptide is associated with mortality in older functionally impaired patients. J Am Geriatr Soc. 2005;53:1991-5. doi:10.1016/j. jacc.2018.02.071.

18. Omland T, Sabatine MS, Jablonski KA, et al. Prognostic value of B-Type natriuretic peptides in patients with stable coronary artery disease: the PEACE Trial. J Am Coll Cardiol. 2007;50:205-14. doi:10.1016/j.jacc.2007.03.038.

19. Doust JA, Pietrzak E, Dobson A, et al. How well does B-type natriuretic peptide predict death and cardiac events in patients with heart failure: systematic review. BMJ. 2005;330:625. doi:10.1136/bmj.330.7492.625.

20. Shang C. B-type natriuretic peptide-guided therapy for perioperative medicine? Open Heart 2014; 1:e000105. doi:10.1136/ openhrt-2014-000105.

21. Gibson SC, Payne CJ, Byrne DS, et al. B-type natriuretic peptide predicts cardiac morbidity and mortality after major surgery. $\mathrm{Br} \mathrm{J}$ Surg. 2007;94. doi:903-910.1002/bjs.5690.

22. Ford MK, Beattie WS, Wijeysundera DN. Systematic review: prediction of perioperative cardiac complications and mortality by the revised cardiac risk index. Ann Intern Med. 2010;152:26-35. doi:10.7326/0003-4819-152-1-201001050-00007.

23. Yun KH, Jeong MH, Oh SK, et al. Preoperative plasma N-terminal pro-brain natriuretic peptide concentration and perioperative cardiovascular risk in elderly patients. Circ J. 2008;72:195-9. doi:10.1253/circj.72.195.

24. Choi JH, Cho DK, Song YB, et al. Preoperative NT-proBNP and CRP predict perioperative major cardiovascular events in non-cardiac surgery. Heart. 2010;96:56-62. doi:10.1136/ hrt.2009.181388.

25. Toscano FV, Apinis A, Leff JD. Pre-operative Risk Stratification Update for Cardiac and Major Vascular Surgery. Curr Anesthesiol Rep. 2015;5:445-51. doi:10.1007/s40140-015-0136-3.

26. Cuthbertson BH, Amiri AR, Croal BL, et al. Utility of B-type natriuretic peptide in predicting perioperative cardiac events in patients undergoing major non-cardiac surgery. $\mathrm{Br} \mathrm{J}$ Anaesth. 2007;99:170-6. doi:10.1093/bja/aem158. 
27. Kistorp C, Raymond I, Pedersen F, et al. N-terminal probrain natriuretic peptide, $\mathrm{C}$-reactive protein, and urinary albumin levels as predictors of mortality and cardiovascular events in older adults. JAMA. 2005;293:1609-16. doi:10.1001/ jama.293.13.1609.

28. McKie PM, Cataliotti A, Lahr BD, et al. The prognostic value of $\mathrm{N}$-terminal pro-B-type natriuretic peptide for death and cardiovascular events in healthy normal and stage $A / B$ heart failure subjects. J Am Coll Cardiol. 2010;55:2140-7. doi:10.1016/j. jacc.2010.01.031.

29. Daniels LB. Natriuretic peptides and assessment of cardiovascular disease risk in asymptomatic persons. Curr Cardiovasc Risk Rep. 2010;4:120-7. doi:10.1007/s12170-010-0078-8.

30. Fleisher LA, Beckman JA, Brown KA, et al. 2009 ACCF/AHA focused update on perioperative beta blockade incorporated into the ACC/AHA 2007 guidelines on perioperative cardiovascular evaluation and care for noncardiac surgery. J Am Coll Cardiol. 2009;54:e13-8. doi:10.1016/j.jacc.2009.07.010.

31. Wolf PA, Abott RD, Kannel WB. Atrial fibrillation: a major contributor to stroke in the elderly. Arch Intern Med. 1987;147:1561-4.

32. Hart RG, Sharma M, Mundl $H$, et al. Rivaroxaban for stroke prevention after embolic stroke of undetermined source. N Engl J Med. 2018;378:2191-201. doi:10.1056/NEJMc1809065.

33. McIntyre WF, Connolly SJ, Healey JS. Atrial fibrillation occurring transiently with stress. Curr Opin Cardiol. 2018;33(1):58-65. doi:10.1097/HCO.0000000000000475.

34. McIntyre WF, Healey JS. Stroke prevention for patients with atrial fibrillation: beyond the guidelines. J Atr Fibrillation. 2017;91(6):1-7. doi:10.4022/jafib.1475.

35. Gialdini G, Nearing K, Bhave PD, et al. Perioperative atrial fibrillation and the long-term risk of ischemic stroke. JAMA. 2014;312(6):616-22. doi:10.1001/jama.2014.9143.

36. Simmers $D$, Potgieter $D$, Ryan $L$, et al. The use of preoperative B-type natriuretic peptide as a predictor of atrial fibrillation after thoracic surgery: systematic review and meta-analysis. J Cardiothorac Vasc Anesth. 2015;29:389-95. doi:10.1053/j. jvca.2014.05.015.
37. Rodseth RN, Biccard BM, Chu R, et al. Postoperative B-type natriuretic peptide for prediction of major cardiac events in patients undergoing noncardiac surgery: Systematic review and individual patient meta-analysis. Anesthesiology. 2013;119:27083. doi:10.1097/ALN.0b013e31829083f1.

38. Cardinale D, Colombo A, Sandri MT, et al. Increased perioperative $\mathrm{N}$-terminal pro-B-type natriuretic peptide levels predict atrial fibrillation after thoracic surgery for lung cancer. Circulation. 2007;115:1339-44. doi:10.1161/ CIRCULATIONAHA.106.647008.

39. Cardinale D, Sandri MT, Colombo A, et al. Prevention of Atrial Fibrillation in High-risk Patients Undergoing Lung Cancer Surgery: The PRESAGE Trial. Ann Surg. 2016;264:244-51. doi:10.1097/ SLA.0000000000001626.

40. Wazni OM, Martin DO, Marrouche NF, et al. Plasma B-type natriuretic peptide levels predict postoperative atrial fibrillation in patients undergoing cardiac surgery. Circulation. 2004;110:124-7. doi:10.1161/01.CIR.0000134481.24511.BC.

41. Nojiri T, Maeda H, Takeuchi $Y$, et al. Predictive value of B-type natriuretic peptide for postoperative atrial fibrillation following pulmonary resection for lung cancer. Eur J Cardiothorac Surg. 2010;37:787-91. doi:10.1016/j.ejcts.2009.09.043.

42. Yoshimura $\mathrm{M}$, Mizuno $\mathrm{Y}$, Nakayama $\mathrm{M}$, et al. B-type natriuretic peptide as a marker of the effects of enalapril in patients with heart failure. Am J Med. 2002;112:716-20. doi:10.1016/S00029343(02)01121-X.

43. Cai GL, Chen J, Hu CB, et al. Value of plasma brain natriuretic peptide levels for predicting postoperative atrial fibrillation: A systemic review and meta-analysis. World J Surg. 2014;38:51-9. doi:10.1007/s00268-013-2284-2.

44. Fabiani I, Colombo A, Bacchiani G, et al. Incidence, Management, Prevention and Outcome of Post-Operative Atrial Fibrillation in Thoracic Surgical Oncology. J Clin Med. 2019;9(1):37. doi: $10.3390 / \mathrm{jcm} 9010037$. 